\title{
Timed Automata Model for Component-Based Real-Time Systems
}

\author{
Georgiana Macariu and Vladimir Creţu \\ Computer Science and Engineering Department \\ Politehnica University of Timisoara \\ Timisoara, Romania \\ Email: georgiana.macariu@cs.upt.ro,vladimir.cretu@cs.upt.ro
}

\begin{abstract}
One of the key challenges in modern real-time embedded systems is safe composition of different software components. Formal verification techniques provide the means for design-time analysis of these systems. This paper introduces an approach based on timed automata for analysis of such component-based real-time embedded systems. The goal of our research is to provide a method for treating the schedulability problem of such systems on multi-core platforms. Since the components are developed, analyzed and tested independent of each other, the impact of one component on the others does not depend on its internal structure. Therefore, we reduce the problem of proving the schedulability of the composed system to proving the schedulability of each component on the resource partition allocated to it based on the interface of the component. The proposed verification method is demonstrated on a H.264 decoder case study.
\end{abstract}

Keywords-real-time scheduling; model checking; components

\section{INTRODUCTION}

Nowadays, real-time embedded software development is focusing more and more on how to build flexible and extensible systems. Component-based software systems achieve this objective by gluing individually designed software components, each component with its own timing requirements. The compositional design of real-time systems can be done using hierarchical scheduling and schedulability analysis of the composed system can be addressed based on component interfaces that abstract the timing requirements of each component. Furthermore, the rapid developments in multiprocessor technology determined a growing interest in multiprocessor scheduling theories and, consequently also in multiprocessor hierarchical scheduling theories [1], [2].

In this context, providing formal guarantees on the schedulability of such component-based systems running on multi-core platforms becomes even more important as these components consist of interacting tasks and each component can have a different scheduling strategy. In this paper we address the formal verification of schedulability of realtime multi-core component-based systems assuming that the components consist of preemptable periodic tasks. These tasks can be independent or there may exist various precedence constraints between them, represented as task graphs. Stopwatch automata [3] have been proposed for modeling of preemptive tasks, but reachability of composition of these automata is undecidable [4]. Moreover, it has been shown that many preemptive multiprocessor scheduling algorithms for periodic tasks suffer of scheduling anomalies when there is a change in their execution time [5]. Therefore, an accurate analysis of systems using these algorithms must consider variable task execution times. This poses another problem to our formal verification of schedulability since it was proved [6] that the schedulability problem on task graphs is undecidable if the following conditions are both met: (1) the scheduling strategy is preemptive and, (2) tasks have variable execution times ranging over a continuous interval.

We propose a formal method for checking the schedulability of real-time component-based applications running on multi-core platforms. Our method uses the timed automata [7] formalism, for which the reachability problem is decidable. Our timed automata model is actually the model of a level in a multi-core scheduling hierarchy. The proposed method uses a discrete time formalism but is also able to capture continuous task execution times by approximating the stopwatch automata model. We show this is true by proving that the formal language accepted by the timed automata model is included in the language accepted by the stopwatch automata and we evaluate the approximation errors. Also, we show how our model can be applied iteratively to check the entire scheduling hierarchy.

Related work. Formal verification of component-based systems is addressed by several frameworks for various purposes. The Save Integrated Development Environment (SAVE-IDE) [8] offers support not only for design of component based systems, but also allows specification of the behavior of each component using timed automata. Using UPPAAL [9] and timed automata models, it is possible to check if the components satisfy their specification. However, the verification features of the IDE do not allow specification of component-level scheduling strategies based only on component interfaces. Ke et. al. [10] also propose a methodology for formal verification of the timing and reactive behavior of component-based systems. Unlike the work presented in this paper, their approach assumes that tasks associated to a component execute on a single processor and each task is modeled by a separate timed automaton. In our case, the timed automata network which models a component uses a different approach in which a 
single automaton is used for all tasks of the component and thus better performance can be achieved. An approach to modeling real-time systems resembling ours is taken in the TIMES tool [11] but until now the tool only offers support for analyzing uniprocessor systems.

Multi-processor schedulability analysis using modelchecking has been investigated in [12]. The models in [12] allow restricted and full migration of task instances. Their work, like the one described in this paper also uses a discrete time formalism, but tasks are assumed to be independent and every task is modeled separately. Task schedulability is checked in decreasing order of task priority which implies that for a task set with $N$ tasks, model checking has to be performed $N$ times in order to determine the schedulability of the entire set. With this approach a maximal number of $N+1$ clocks are necessary for a task set of size $N$. Unlike this model checking solution, our proposal requires just a single run of the model checking for the entire task set using a single clock.

Madl et al. [13] introduce model checking for schedulability analysis of preemptive event-driven asynchronous distributed real-time systems with execution intervals. The analysis method proposed here starts from the same essential idea as the work in [13] but there are several significant differences. First, we assume a hierarchical scheduling model which means that the execution of tasks is constrained by the availability of the temporal partitions. Second, unlike the work in [13] which assumes tasks are partitioned between processors, task migration is allowed in our model. Last, instead of modeling just the tasks of an application individually, we model a whole scheduling level.

\section{Preliminaries on Timed Automata}

In this section we give basic descriptions and definitions related to the timed automata used in our work.

Formal syntax. Assume a finite set of real-valued clocks $\mathcal{C}$ and $\mathcal{B}(\mathcal{C})$ the set of constraints on the clocks in $\mathcal{C}$. The clock constraints (guards) are conjunctions of expressions of the form $x \bowtie N$ and $x-y \bowtie N$ where $x, y \in \mathcal{C}, N \in \mathbb{N}$ and $\bowtie \in\{<, \leq,=, \geq,>\}$. A timed automaton over the set of clocks $\mathcal{C}$ is a tuple $\left\langle L, l_{0}, \Sigma, \mathcal{C}, I, E\right\rangle$ where

- $\mathrm{L}$ is a set of finite locations,

- $l_{0}$ is the initial location,

- $\Sigma$ is a set of actions,

- $\mathcal{C}$ is the set of clock variables,

- $I: L \rightarrow \mathcal{B}(\mathcal{C})$ associates invariants to locations,

- $E \subseteq L \times \mathcal{B}(\mathcal{C}) \times \Sigma \times 2^{\mathcal{C}} \times L$ is the set of transitions, where transition $\left\langle l, g, a, r, l^{\prime}\right\rangle$ from location $l$ to location $l^{\prime}$, labeled with action $a$ is executed only if guard $g$ is true and resets clocks in $r \subseteq \mathcal{C}$.

All timed automata models presented in this paper are based on the UPPAAL [9] model of timed automata which is extended with constructs such as constants, integers, committed and urgent constraints on locations, networks of timed automata and events transmitted between automata. An urgent location is similar to a location with all incoming transitions resetting a clock $x$ and having associated an invariant $x \leq 0$ (i.e. time cannot pass while the automaton is in an urgent location).

Semantics. For a timed automaton we can define a clock valuation function $v: \mathcal{C} \rightarrow \mathbb{R}_{+}$assigning positive real values to clocks in $\mathcal{C}$. A state $s$ in the timed automaton is a pair $(l, v)$ where $l \in L$ and $v$ is a clock valuation. The automaton can stay in state $s$ as long as the invariant associated to $l$ is true or can execute transitions outgoing from $l$ when the guard of these transitions is true. Therefore, two types of transitions can be defined:

- delay transitions: $(l, v) \stackrel{d}{\rightarrow}\left(l, v^{\prime}\right)$ where $v^{\prime}(x)=v(x)+$ $d, \forall x \in \mathcal{C}$ and $v^{\prime}$ preserves the invariant of location $l$,

- action transitions: $(l, v) \stackrel{a}{\rightarrow}\left(l^{\prime}, v^{\prime}\right)$ if there exists a transition $\left\langle l, g, a, r, l^{\prime}\right\rangle \in E$ and guard $g$ is true for clock valuation $v$ and $v^{\prime}$ is obtained from $v$ by resetting all clocks in $r \subseteq \mathcal{C}$ and leaving all others unchanged.

Networks of timed automata. A network of $n$ timed automata $A_{i}=\left\langle L_{i}, l_{i}^{0}, \Sigma, \mathcal{C}, I_{i}, E_{i}\right\rangle, 1 \leq i \leq n$ over a common set of clocks and actions is a parallel composition of $A_{i}$, describing a timed automaton obtained from its component automata. Semantically, the network of timed automata requires joint execution of delay transitions and synchronization over complementary action transitions.

For networks of timed automata, UPPAAL introduces the concept of committed locations. A committed location is more restrictive than an urgent location, as a state containing a committed location cannot delay and the next transition of the system must involve an outgoing edge from one of the committed locations in the state.

\section{SySTEM MODEL}

\section{A. Real-Time Components and Component Contracts}

In this paper we assume that real-time software applications consist of independent components. Further, each component consists of a set of independent multi-threaded tasks (MTTs), where each MTT is made up of periodic tasks with execution costs defined as continuous intervals but with a common period and deadline. The tasks in an MTT may run in parallel and it is also possible to define precedence constraints between them. The execution patterns of all tasks of a component are modeled using a timed automaton as it is described in the next sections.

Definition 1 (Component). A component $C$ consists of a finite set $\mathcal{M T}$ of $n$ MTTs where:

- a MTT $\Theta_{i} \in \mathcal{M T}$, with $1 \leq i \leq n$, is a tuple $\Theta_{i}=$ $\left(\mathcal{T}_{i}, p_{i}, d_{i}, r_{i}\right)$, where $\mathcal{T}_{i}$ is the set of $t_{i}$ tasks in the MTT, $p_{i}$ represents the inter-arrival time between different instances of the same MTT, $d_{i}$ is the deadline by which all tasks in $\mathcal{T}_{i}$ should finish and $r_{i}$ represents the time of the first release of $\Theta_{i}$, 
- each task $\tau_{j} \in \mathcal{T}_{i}, 1 \leq j \leq t_{i}$, is characterized by a tuple $\left(\right.$ bcet $_{j}$, wcet $_{j}$, prio $\left._{j}\right)$ where bcet ${ }_{j}$ and wcet $_{j}$ are integer values that specify the limits of the continuous execution interval of task $\tau_{j}$ and prio $_{j}$ is the priority of the task.

All numeric parameters in Definition 1 are considered integer numbers.

The tasks belonging to each component are scheduled separately using a component-specific preemptive scheduling policy. Therefore, when building an application based on such components one must ensure that the tasks of each component are schedulable independent of the execution of any other component in the application.

One of the solutions for ensuring temporal isolation of components running on uni-processor or multi-processor real-time systems is provided by hierarchical scheduling schemes based on execution time servers [14]. In hierarchical scheduling each application has its own scheduler and can use the scheduling policy that best suits its needs. Based on such a hierarchical scheduling scheme, Harbour has introduced the concept of service contracts [15]. In Harbour's model, every application or application component may have a set of service contracts describing its minimum resource requirement. Similarly, we define service contracts to capture the resource requirements of our components. Next, each service contract is mapped to a set of execution time servers which mark the limits of the resources allocated by the application to the child component. An execution time server in a multi-core system, as considered in this paper, is characterized by a tuple $(Q, P)$ meaning that the component will receive $Q$ units of execution every $P$ units of time. Additionally, we consider a third parameter $o$ representing the time when the server is first released. It is assumed there is a finite set of servers $\mathcal{S}$ containing the servers for all components of an application.

In terms of the timed automata formalism we define a component contract as follows:

Definition 2 (Component contract). A component contract $\mathcal{C}_{C}$ providing a set of $n_{s}$ execution servers $\mathcal{S}_{C} \subseteq \mathcal{S}$ is a timed automaton $\mathcal{A}_{C c}$ over the set of actions $\Sigma_{\mathcal{C}_{\mathcal{C}}}$ such that:

- $\mathcal{A}_{C c}$ specifies the activation pattern of servers $\sigma_{i}=$ $\left(Q_{i}, P_{i}, o_{i}\right) \in \mathcal{S}_{C}, 1 \leq i \leq n_{s}$,

- $\Sigma_{\mathcal{C}_{\mathcal{C}}}=\{$ active, inactive $\}$, where action active signals to the component scheduler that a server $\sigma_{i} \in \mathcal{S}_{C}$ has just become active (i.e. the processor is now available to be used by the component), while inactive signals deactivation of the server.

We consider that parameters $Q_{i}, P_{i}$ and $o_{i}$ have integer values.

The component also has associated a scheduler which will schedule for execution the tasks of the MTTs in a component according to a preemptive scheduling policy. We consider that a global scheduling policy is used and, as such, a task can run on any processing unit. As a consequence, task migration may occur whenever a task is preempted or suspended. The scheduler of the component is modeled by a timed automaton with the following characteristics:

- has a queue holding the tasks ready for execution,

- implements a preemptive scheduling policy representing a sorting function for the task queue,

- maintains a map between active execution servers and tasks using the servers, and

- has an Error location which is reached when a task misses its deadline.

A component consisting of $n$ MTTs could have been modeled also using a timed automaton for each of the tasks of the MTTs, each automaton with its own clock. Since the state space of timed automata models grows exponentially with the number of clocks in the model, we decided to build a single timed automaton which models the execution patterns of all $n$ MTTs and reduce the number of clocks to one as it will be shown in the following subsection. Moreover, each task could have been modeled using stopwatch automata but the reachability analysis of composed stopwatch automata is undecidable [4]. The same observation applies for modeling the component as a single stopwatch automaton and consequently, we propose an approximation of a stopwatch model using timed automata with discrete clocks to keep track of the execution time of each task.

\section{B. Timed Automata Model for Real-Time Components}

In the timed automata formalism a component of a realtime application is the network of timed automata obtained through parallel composition of the automaton which models the execution pattern of the MTTs of the component, the component scheduler automaton and the timed automaton modeling the activation and deactivation patterns of the execution time servers (i.e. the $\mathcal{A}_{C c}$ automaton in Definition 2). In what follows we will use the names Task Generator (TGT) to denote the timed automaton which models the MTTs' execution and Server Generator ( $S G$ ) for the one modeling the servers. Apart from these, the network also includes a Timer automaton which uses a single continuous clock $t$ and each time this clock ticks the automaton sends a tick signal to the $T G T$ and the $S G$ automata.

Before explaining in more detail the timed automata model we introduce some notations. For each MTT $\Theta_{i}$ we use a variable $R(i)$ to hold the time of the next release of $\Theta_{i}$. In order to determine the actual execution time of each task $\tau_{j}$, we use a variable $E(j)$ to keep track of the time task $\tau_{j}$ has executed since its last release. Basically, $E(j)$ acts like a discrete clock which can be suspended and resumed. Also, for each task $\tau_{j}$ a variable status $(j)$ indicates its current status and is initialized to idle meaning that a task instance has not been released yet. The value $\operatorname{status}(j)=$ ready is used to denote that a task instance of 


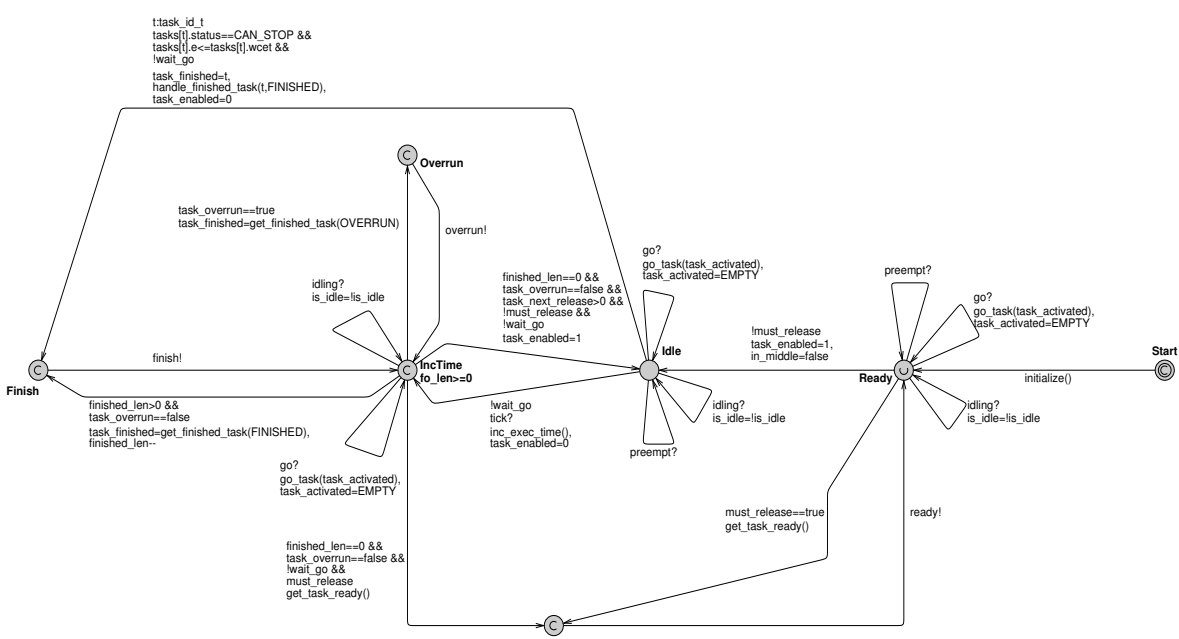

(a) The Task Generator timed automaton.

Figure 1. Task and Server generators.

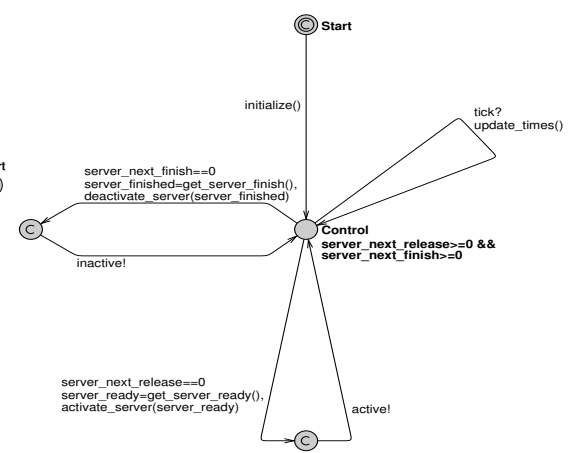

(b) The Server Generator timed automaton. $\tau_{j}$ is ready for execution (i.e. it has just been released or was preempted). If $\tau_{j}$ is waiting for one of its predecessor tasks to finish then status $(j)=$ waiting. If an instance of task $\tau_{j}$ is running then status $(j)=$ running. A task $\tau_{j}$ which has executed for $b_{c e t}$ time units but for less than $w_{c e t}$ units will have status $(j)=$ can_stop. To denote that an instance of task $\tau_{j}$ has finished or has missed its deadline we use $\operatorname{status}(j)=$ finished and $\operatorname{status}(j)=$ overrun, respectively. The discrete clock $E(j)$ keeps track of the overall time for which status $(j)=$ running.

The TGT automaton presented in Figure 1(a) uses a variable task next release to remember the time until one of its MTTs must be released and a variable task_next_finish to keep the earliest time when one of the currently running tasks should finish. At start-up, task_next_release is initialized to $\min (R(i)), i=1,2, \ldots, n$ and the automaton goes to the Ready location. From this location, if task_next_release $=0$ and there is at least one task $\tau_{j}$ with $\operatorname{status}(j)=i d l e$ (i.e. $T G T$ must release a task), the automaton executes the transition with the guard must_release $=$ true and in function get_ready_task () elects the task $\tau_{j}, j=1,2, . ., t_{i}$, for which status $(j)=i d l e$, updates task_next_release (only if this is the first task $\tau_{j}$ in the MTT that is released in the current period) and task_next_finish, sets a shared variable task_ready to $(i-1) \cdot n+j$ (a global identifier of task $\tau_{j}$ of the MTT $\Theta_{j}$ ) and then sends the ready signal to the scheduler automaton which will read the task_ready variable and will add task $\tau_{j}$ to its queue. The process is repeated until task_next_release becomes greater than 0 and there are no idle tasks for any MTT that has just been released. At this point the automaton goes to the Idle location where it waits for a tick signal from the Timer automaton. On each release of the MTT $\Theta_{i}, R(i)$ is postponed with $p_{i}$.

The $S G$ automaton presented in Figure 1(b) works in a similar way with the distinction that generated active servers are continuous (not preemptable). For each server $\sigma_{k}, 1 \leq k \leq n_{s}$, there is a discrete clock $R E(k)$ analogous to $E(j)$ and a variable $R R(k)$ is used to keep the time until the next activation of $\sigma_{k}$. Two additional variables, server_next_release and server_next_finish hold the time until the earliest start time of a server $\sigma_{k}$ and the earliest finish time, respectively. When server_next_release $=0$ a processing unit becomes available for the component (i.e. some $\sigma_{k}$ starts) and the $S G$ takes the transition guarded with server_next_release $=0$. In function get_sever_ready () $S G$ determines the server $\sigma_{k}$ which became active, updates server_next_release and server_next_finish and sets a shared variable server_ready to $k$. Afterwards, the active signal is sent to the scheduler of the component to announce the activation of server $\sigma_{k}$. Also, for the server that just started, $R R(k)$ is set to $P_{k}$. When $\sigma_{k}$ finishes and the processing unit is no longer available, the automaton takes the transition guarded with server_next_finish $=0$ and, similar to the previous scenario, sets the shared variable server_finished $=k$ and sends the inactive signal to the scheduler.

On every tick of the timer, TGT leaves the Idle location and goes to the IncTime location. During this transition, in function inc_exec_time(), the current execution time $E(j)$ of all tasks $\tau_{j}$ running (with status set to running or can_stop) at that time are increased with a value $M I \bar{N}$ representing the minimum between task_next_release, task_next_finish, server_next_release and server_next_finish. If, as a result of this update, there are tasks for which $E(j)$ reached bcet $_{j}$ then we set $\operatorname{status}(j)=$ can_stop and if $E(j)=$ wcet $_{j}$ then the task has finished its execution, status $(j)$ becomes finished and a variable finished_len counting the finished tasks is incremented. At the same time, we identify any task $\tau_{j}$ that missed its deadline and set $\operatorname{status}(j)=$ overrun. Also, as time passes the time $R(i)$ 
of the next release of each MTT $\Theta_{i}$ is decreased with $M I N$ and the values task_next_release and task_next_finish are updated. When the $S G$ receives the tick signal from the Timer, in function update_times(), it increases the current activation length $R E(k)$ of all active servers $\sigma_{k}$ with $M I N$ and decreases $R R(k)$ of all servers with the same value. Also the values of the variables server_next_release and server_next_finish are updated.

If some $R(i)$ reaches 0 then a new instance of the MTT is released (TGT sends the ready signal to the scheduler as explained before). When the scheduler (see Figure 2) receives notification of a new task being released, it checks if a server on which to schedule the task is available and, if so, sends the go signal to the $T G T$ automaton and sets the entry in its server-task map accordingly. If the priority of the newly released task is higher than the priority of one of the running tasks and no active servers are idle, the scheduler will preempt the lower priority task and will give the server to the higher priority task. If no server is available or the server is deactivated while a task is running on it, the task is either scheduled on another server (if its priority allows it) or is queued. On every tick the $T G T$ automaton searches for all tasks $\tau_{j}$ that finished their execution or that missed their deadline and sends finish or overrun signals to the scheduler. If the server used by a finished task is still active and there are ready tasks waiting in the scheduler's queue, a new task is started and the go signal is sent to TGT. Moreover, when an active server $\sigma_{k}$ finishes, the scheduler will attempt to reschedule the task that was using the server associated with it on some other free server available to the component. If no active server is free, then the lowest priority running task may be preempted. Between all automata, data (e.g. task identifier or resource identifier) is transmitted using shared variables. A more detailed description of the scheduler timed automaton can also be found in [16].

In order to be able to capture task execution intervals in continuous time, when the TGT automaton is in the Idle location, if there is at least one task $\tau_{j}$ with $\operatorname{status}(j)=$ can_stop, the automaton may decide non-deterministically to finish the task. A remark that must be made is that whenever there is at least one task with status can_stop the value of $M I N$ is set to 1 . This implies that at the next tick signal, the discrete clocks presented above are increased with a single time unit. If a task $\tau_{j}$ finishes at some fraction of the time unit, another task $\tau_{l}$ that was previously preempted or is ready to be released may take the place of $\tau_{j}$. However, because in this case we cannot keep track in the discrete clock $E(l)$ of the time task $\tau_{l}$ is executing until the first tick after it has been started/restarted, the value in $E(l)$ is only an approximation of the real execution time of $\tau_{l}$. Although, this approach represents just an approximation model of the real system, we will show in the next section that the model preserves the properties of the system and any component that is deemed schedulable with our model is indeed schedulable.

It is important to notice that once each component of an application is proved to be schedulable, by using reachability analysis on our model we can also check the schedulability of the entire application as follows. Each execution time server $\sigma_{k}$ is basically a periodic task with hard deadlines and fixed execution requirement $Q_{k}$. Therefore it can be considered as an MTT consisting of a single task with bcet = wcet and the whole application can be seen as just another component with its own scheduler and whose MTTs are the execution servers corresponding to the service contracts of its components. If we consider that the application also has a service contract mapped to another set of execution server we can again check the proposed model by changing only the parameters of the MTTs and of the execution servers to reflect the new scheduling level represented by the parent application.

\section{Analysis of the Timed Automata Model APPROXIMATION}

A. Stopwatch Automata as a Model for Real-Time Components

Stopwatch automata [3] can be defined as timed automata for which clocks can be stopped and later resumed with the same value. These clocks are called stopwatches and provide a simple way for modeling preemptive real-time tasks. Syntactically, a stopwatch automaton SWA is a tuple $\left\langle L, l_{0}, \Sigma, \mathcal{C}, I, E, A\right\rangle$ where $L, l_{0}, \Sigma, \mathcal{C}, I, E$ have the same meaning as for timed automata (see Section II) and $A$ : $L \times \mathcal{C} \rightarrow\{0,1\}$ is a function that defines the rates of clocks $c_{i} \in \mathcal{C}$ in locations as differential functions $\dot{v}\left(c_{i}\right)=k_{i}$ where $k_{i} \in\{0,1\}$.

From a semantical point of view, the element that distinguishes the SWA from the timed automaton is the clock valuation function $v: \mathcal{C} \rightarrow \mathbb{R}_{+}$assigning positive real values to clocks in $\mathcal{C}$. In a SWA the value of a clock variable during a delay transition $(l, v) \stackrel{d}{\rightarrow}\left(l, v^{\prime}\right)$ is updated to $v^{\prime}\left(c_{i}\right)=v\left(c_{i}\right)+A\left(l, c_{i}\right) \cdot d, \forall c_{i} \in \mathcal{C}$.

In our case, since the tasks belonging to the MTTs of a component are scheduled using a preemptive scheduling policy we could have chosen to model the component using the stopwatch automaton in Figure 3. The execution time of each task in each MTT is represented as a stopwatch clock $e c_{j}, \forall j \in\left\{1,2, \ldots, n \cdot t_{i}\right\}$ with $1 \leq i \leq n$. With each MTT we associate a clock $d c_{i}, \forall i \in\{1,2, . ., n\}$ which will keep track of the MTT's deadline.

In the stopwatch version of our components we only need to replace the TGT automaton with a Task Generator stopwatch automaton (TGS). The TGS presented in Figure 3 still uses the variable task_next_release to remember the time until one of its MTTs must be released but it is not necessary to keep the variable task_next_finish. When the system starts, task_next_release is initialized to 


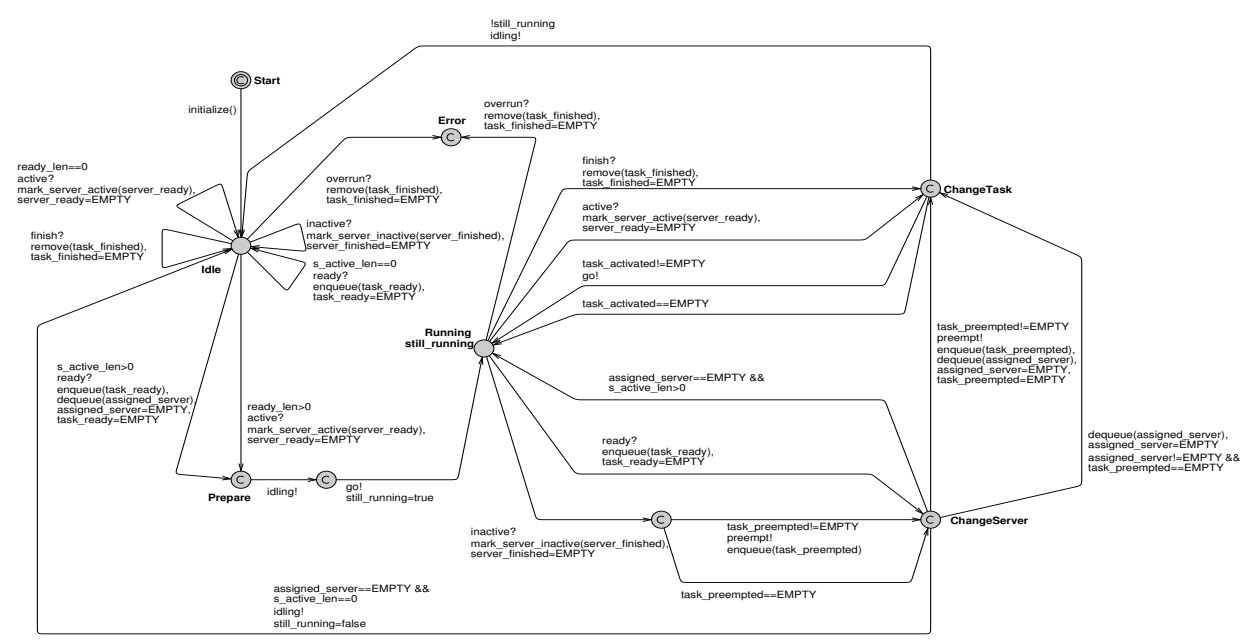

Figure 2. Timed automaton model for the scheduler of the component.

$\min (R(i)), i=1,2, . ., n$ and the automaton goes to the Ready location. From this location, it can either go the Idle location if there are no tasks ready for release or, if there is at least one task $\tau_{j}$ with $\operatorname{status}(j)=i d l e$, the automaton executes the transition with the guard must_release $=$ true and, in function get_ready_task() elects the task $\tau_{j}, j=$ $1,2, . ., t_{i}$, which is idle, updates task_next_release, puts the task global identifier $(i-1) \cdot n+j$ in the shared variable task_ready and sends the ready signal to the scheduler automaton. The scheduler will read the task_ready variable and will add task $\tau_{j}$ to its queue. On each release of the MTT $\Theta_{i}, R(i)$ is postponed with $p_{i}$. After all tasks that are ready to start are released the stopwatch automaton goes to the Idle location where it waits for a tick signal from the Timer automaton or for a running task $\tau_{j}$ to finish. Another event which may take the automaton out of the Idle location is a missed deadline of any MTT. The rates of the clocks $e c_{j}$ and $d c_{i}$ are specified in the guard of the Idle location: $d c_{i}^{\prime}=1$ for all MTTs which contain at least one task that is not finished yet, otherwise $d c_{i}^{\prime}=0$ and $e c_{j}^{\prime}=1$ for all tasks that have $\operatorname{status}(j)=$ running but $e c_{j}^{\prime}=0$ for the other tasks.

In the stopwatch automaton the execution time of each task $\tau_{j}$ is measured by stopwatch $e c_{j}$ started at the release of the task, when the automaton sends the ready signal to the scheduler automaton while the variable task_ready $=j$, until the task finishes and the finish signal is sent with variable task_finished $=j$. $e c_{j}$ does not include the time while the task was preempted. Therefore, for any task $\tau_{j}$ belonging to the multi-threaded task $\Theta_{i}$ the following constraints should be true such that we can say that $\tau_{j}$ has not missed its deadline:

$$
0 \leq e c_{j} \leq \text { wcet }_{j}, 0 \leq e c_{j} \leq d c_{i}, 0 \leq \text { bcet }_{j} \leq \text { wcet }_{j}
$$

Definition 3. A multi-threaded task $\Theta_{i}=\left(\mathcal{T}_{i}, p_{i}, d_{i}, r_{i}\right)$ is schedulable iff all its tasks $\tau_{j}=\left(\right.$ bcet $_{j}$, wcet $_{j}$, prio $\left._{j}\right) \in \mathcal{T}_{i}$ finish execution before the deadline of $\Theta_{i}: d c_{i} \leq d_{i}$ when $e c_{j}=$ wcet $_{j}, \forall j \in\left\{1,2, \ldots, t_{i}\right\}$.

Definition 4. A component is schedulable iff all its multithreaded tasks are schedulable.

The set of actions of the TGS is $\Sigma=$ \{ready,go,preempt,finish,overrun,idling,tick\}. The idling signal is sent by the scheduler automaton when it goes in or out of the Idle location. TGS stays in the Idle location as long as either there is no server active or there are no ready tasks to be scheduled or both of these conditions are true. The go and preempt signals are controlled also by the scheduler. The stopwatch automaton will send ready for every new release of a task instance and finish at its end.

A timed word over the alphabet $\Sigma$ is a pair $(\rho, \theta)$ where $\rho=\rho_{1}, \rho_{2}, .$. is an infinite sequence of events in $\Sigma$ and $\theta=\theta_{1}, \theta_{2}, .$. is a timed sequence denoting the timestamps of the events in $\rho$. A timed language over $\Sigma$ is a set of timed words over $\Sigma$. The timed language $L(S)$ accepted by the stopwatch automaton is the union of the timed languages $L_{j}(S)$ where the words in each language $L_{j}(S)$ refer to valid event sequences generated during the execution of task $\tau_{j}$. We consider that $L(S)=\bigcup_{1 \leq i \leq n} \bigcup_{1 \leq j \leq t_{i}} L_{j}(S)$ because the semantics of task related events in $\Sigma$ are established only in correspondence with a shared variable indicating the task to which the event refers. The untimed words in all $L_{j}(S)$, and consequently in $L(S)$, are described by the following regular expression:

$$
E_{S}=\left(\text { ready,go, }(\text { preempt }, g o)^{*}, \text { finish }\right)
$$

In our case the timestamps of all events $\{$ ready,go,preempt,finish $\}$ acceptable by the stopwatch automaton have to be less than the deadline of the MTT containing the task for which the event appeared (i.e. the task is indicated in a shared variable). This implies 


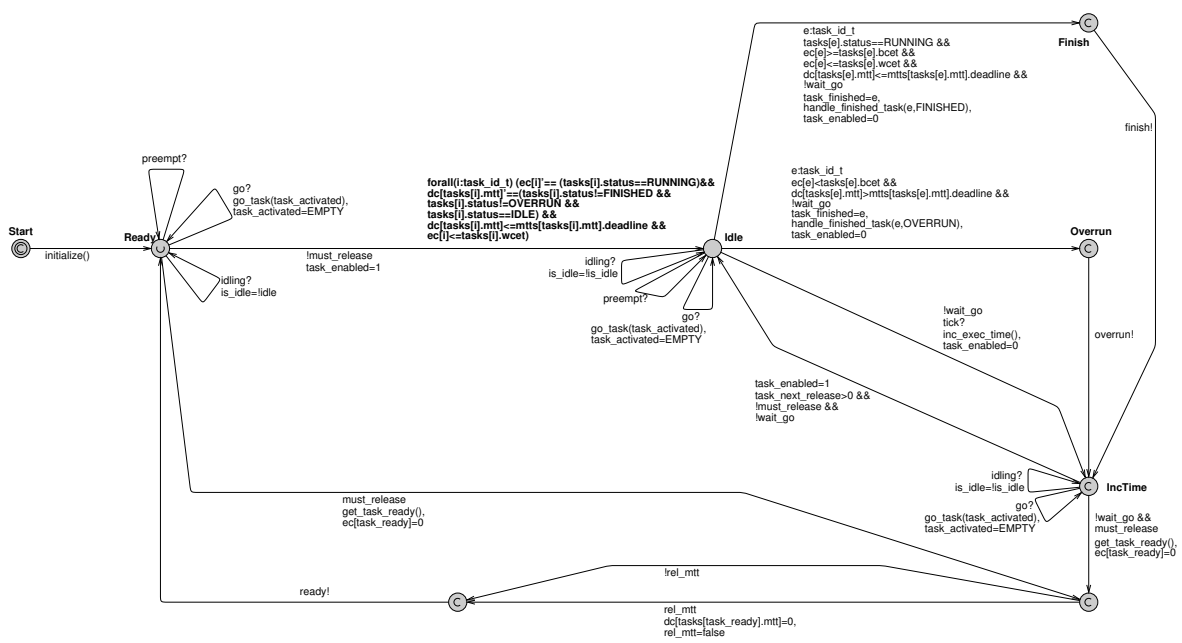

Figure 3. Stopwatch automata model of a real-time component.

that the time a task $\tau_{j}$ in $\Theta_{i}$ spends in the ready state, denoted from now as $T_{\text {ready }}$, must be lower or equal than $d_{i}-$ wcet $_{j}-T_{\text {wait }_{j}}$, where $T_{\text {wait }_{j}}$ is the time the task has to wait for its predecessors to finish.

Problem formulation. The $T G S$ is not an initialized stopwatch automaton and consequently reachability is undecidable for it [17]. Moreover, it has been demonstrated in [6] that the schedulability analysis problem for multiprocessor systems is undecidable if (1) the tasks are scheduled using a preemptive scheduling strategy, and (2) the tasks have execution times ranging over a continuous time interval. Next, we show that the timed automata model for component-based applications proposed by us and described in Section III provides a decidable method for verification of real-time component-based applications which approximates the stopwatch automata model with discrete clocks but at the same time is able to capture continuous execution time intervals.

\section{B. Approximation of Components using Timed Automata}

In this section we show what are the approximation errors implied by the proposed method for schedulability analysis of real-time component-based applications. We also prove that any component declared schedulable by our method it would also be declared schedulable by the stopwatch model. We do this by showing that the language $L(T)$ of $T G T$ is also accepted by $T G S$.

The alphabet of the $T G T$ is similar to the one of the stopwatch automaton. The tick and idling events in $\Sigma$ are not directly related to the execution of the task and only help in modeling the discrete time. These two signals are kept in TGS since the activation time of the servers is still measured with discrete clocks (i.e. we see them as non-preemptable tasks with integer parameters). Keeping the discrete clocks also in $T G S$, instead of replacing them with real clocks, has no influence on the accuracy of the model and on the schedulability analysis. Therefore, in what follows we will refer mostly to the task-related events in $\Sigma$ : ready, go, preempt, finish (overrun means the system is not schedulable and, as we analyze the conditions under which the system is schedulable, we assume this event does not appear).

For the proposed discrete time model we have chosen to consider a time unit equal to 1 . The approximation errors in our model arise from the following situations:

(1) a task $\tau_{j}$ starts its execution at some subdivision of the time unit and

(2) a task $\tau_{j}$ resumes its execution (after it has been preempted) at some subdivision of the time unit.

Since we cannot measure the time from the start/restart point of $\tau_{j}$ until the beginning of the next time unit, the subunit of execution time will not be reflected by $E(j)$.

A task $\tau_{j}$ can be preempted either when a higher priority task $\tau_{l}$ becomes ready for execution or the server used by task $\tau_{j}$ is deactivated and there is no other active and free server. Activation and deactivation of servers is observed by $T G T$ only through task preemptions and resumptions. As for all MTTs the release time is an integer value, a higher priority task $\tau_{l}$ can be released for execution while $\tau_{j}$ is executing only at discrete moments. If $\tau_{l}$ does not depend on any task it means that, in this case, $\tau_{l}$ can preempt $\tau_{j}$ only at discrete moments of time and the clocks $E(j)$ and $E(l)$ will behave just like the clocks $e c_{j}$ and $e c_{l}$. If $\tau_{l}$ depends on some task $\tau_{k}$ and $\tau_{k}$ finishes at some time between two consecutive discrete moments then this makes it possible for $\tau_{l}$ to preempt $\tau_{j}$. In the later case, the clocks $E(j)$ and $E(l)$ will not be increased at next discrete time point. If task $\tau_{j}$ resumes is execution during the same fraction of time in which it was preempted, clock $E(j)$ will still not be increased as in this case $\tau_{j}$ 's role is similar to that of $\tau_{l}$ in the previous case. This implies that no matter how many 
times the task is preempted between two successive discrete time points, in the computation of $E(j)$ this will have the same effect as a single preemption. Since all tasks of an MTT $\Theta_{i}$ have the same deadline $d_{i}$ and are released at the same time then for all tasks in $\Theta_{i}$ at most $m_{j}$ preemptions may influence the value of the discrete clock $E(*)$ without a deadline miss occurring, where

$$
1 \leq m_{j} \leq d_{i}-T_{\text {wait }_{j}}, m_{j} \in \mathbb{N}
$$

The alphabet of $T G T$ is the alphabet of the $T G S$, namely $\Sigma=\{$ ready,go,preempt, finish,overrun,idling,tick $\}$. Just like for the stopwatch automaton, in this case also we are interested only in the events in $\Sigma$ related to task execution. Therefore, for each task $\tau_{j}$, the timed automata has to accept timed words following the syntax of the untimed regular expression:

$$
E_{T}=\left(\text { ready, go, }(\text { preempt }, \text { go })^{*}, \text { finish }\right)
$$

We see that $E_{T}=E_{S}$.

In what follows we use $v_{s w_{j}}$ to denote the valuation of the stopwatch clock $e c_{j}$ in $T G S$ and $v_{t}$ for the valuation of the continuous clock in $T G T$, where $v_{t} \in[0,1]$. We also consider a valuation $v_{j}=E(j)+v_{t}$ for each task $\tau_{j}$. This helps in measuring the approximation error of the proposed model. Note that $v_{j}$ is an approximation of $v_{s w_{j}}$. As at most $m_{j}$ preemptions of a task $\tau_{j}$ can influence the value of $E(j)$ it results that $v_{s w_{j}}-v_{j} \leq m_{j}$. Also, we use $v_{d c_{i}}$ to denote the valuation of the clock $d c_{i}$ in $T G S$ and we consider the valuation $v_{\Theta_{i}}=p_{i}-R(i)+v_{t}$ which measures the time since the release of the MTT $\Theta_{i}$. The valuation $v_{\Theta_{i}}$ will grow with the same slope as the valuation $v_{d c_{i}}$ and consequently $v_{\Theta_{i}}=v_{d c_{i}}$ at any time between the release of $\Theta_{i}$ and its finish.

In order to establish the relationship between $T G T$ and $T G S$, we must compare the timed words that follow the syntax of $E_{T}$. We assume the timestamps of these words analyzed in relation to the valuations $v_{\Theta_{i}}$ for $T G T$ and $v_{d c_{i}}$ for $T G S$, respectively, are the same.

Theorem 1. For any timed word that follows the syntax of $E_{T}$ and simulates the execution trace of a task $\tau_{j}$ on both TGT and TGS automata, $v_{s w_{j}}-m_{j} \leq v_{j} \leq v_{s w_{j}}$ holds from the release of the task until its ending, $\forall j \in\left\{1,2, \ldots, t_{i}\right\}$ and $\forall i \in\{1,2, \ldots, n\}$.

Proof: Both TGS and TGT receive events related to a specific task in the same order and with the same timestamps (related to $v_{\Theta_{i}}$ and $v_{d c_{i}}$, respectively). Whenever the $T G S$ receives a go signal it sets the status of the task to running and when it receives the preempt signal the status of the task is set to ready. The behavior of the TGS upon receiving the go and preempt events is the same.

When the status of the task is running, $v_{s w_{j}}$ grows with slope 1 and stays constant when the task has status ready.
The valuation $v_{j}$ also stays constant while the task is in the ready state and grows with slope 1 when the task is running due to the $v_{t}$ component. If the task is preempted only at discrete time points (e.g. when the server that the task was using finished its available execution units) then at the end of the task $v_{j}=v_{s w_{j}}$. However, if a task preemption happens between two successive distinct time points, the valuation $v_{t}$ is not added to $v_{j}$, whereas $v_{s w_{j}}$ will contain also this fraction of time unit, and therefore $v_{j} \leq v_{s w_{j}}$. Since the $v_{j}$ can decrease for at most $m_{j}$ times, each time with at most 1 time unit, it follows that $v_{s w_{j}}-m_{j} \leq v_{j}$.

The inequality in Theorem 1 shows that during the simulation of the same word on both the TGT and TGS automata, it will take at least the same amount of time for $v_{j}$ to reach a specific value as it will take to $v_{s w_{j}}$.

Next we analyze possible timestamps of the finish event. As this event is related to the guards in the automata that contain the best case execution time bcet $_{j}$ of a task $\tau_{j}$ and its worst case execution time wcet $_{j}$ we will determine what is the relation between the actual best execution time $t_{b c e t_{j}}$ and the actual worst execution time $t_{w c e t}$ of $\tau_{j}$ and the valuations $v_{j}=$ bcet $_{j}$ and $v_{j}=$ wcet $_{j}$. Note that $v_{j}$ does not include those fractions of time units that we cannot measure in the $T G T$.

Theorem 2. For any timed word that follows the syntax of $E_{T}$ and simulates the execution trace of a task $\tau_{j}$ on TGT, if $v_{j}=$ bcet $_{j}$ then $t_{\text {bcet }_{j}} \leq$ bcet $_{j}, \forall j \in\left\{1,2, \ldots, t_{i}\right\}$ and $\forall i \in\{1,2, \ldots, n\}$.

Proof: If task $\tau_{j}$ is not preempted during its execution or is preempted only at discrete time points then $t_{b c e t_{j}}=$ bcet $_{j}$. If the task is preempted between two consecutive discrete time points then the valuation $v_{j}$ when the task will resume its execution will not contain the subunit of time that it had executed before it was preempted and only an integer number of time units. In contrast, $t_{b c e t_{j}}$ will contain those time fractions and, therefore it will reach $b_{c e t}$ faster than $v_{j}$ which means that $t_{b c e t_{j}}<b_{c e t}$.

Theorem 3. For any timed word that follows the syntax of $E_{T}$ and simulates the execution trace of a task $\tau_{j}$ on TGT, if $v_{j}=$ wcet $_{j}$ then wcet $_{j} \leq t_{\text {wcet }_{j}}, \forall j \in\left\{1,2, \ldots, t_{i}\right\}$ and $\forall i \in\{1,2, \ldots, n\}$.

Proof: If task $\tau_{j}$ is not preempted during its execution or is preempted only at discrete time points then $t_{w c e t}=$ wcet $_{j}$. If the task is preempted between two consecutive discrete time points then the valuation $v_{j}$ when the task will resume its execution will not contain the subunit of time that it had executed between before it was preempted and only an integer number of time units. In contrast, $t_{w c e t}$ will contain those time fractions and, therefore by the time $v_{j}$ will reach wcet $_{j} t_{w c e t}$ will be greater than wcet $_{j}$.

From theorems 2 and 3 it follows that, if a task finishes its execution before its deadline in $T G T$ it will always meet 
its deadline in $T G S$ which means that if the task is proven schedulable in $T G T$ then it will also be schedulable in $T G S$.

For a task $\tau_{j}$ belonging to a MTT $\Theta_{i}$ to be schedulable it is also required for it to finish before its deadline. If $T_{r e a d y_{j}}$ is the time for which the task has the ready status then we say that the task is schedulable if the following condition is satisfied:

$$
T_{\text {ready }_{j}}+m_{j} \leq d_{i}-\text { wcet }_{j}-T_{\text {wait }_{j}}
$$

The condition 5 says that task $\tau_{j}$ can be preempted for at most $d_{i}-$ wcet $-T_{\text {wait }_{j}}-m_{j}$ before its deadline. The $m_{j}$ term appears due to the imprecision of the model.

Next we prove that $T G S$ accepts the timed language over $\Sigma$ that $T G T$ accepts. Specifically we prove that $L(T) \subseteq$ $L(S)$ by checking the intersection $L(T) \bigcap \overline{L(S)}=\emptyset$. We have already shown that the syntax of the timed language $L(S)=\bigcup_{1 \leq i \leq n} \bigcup_{1 \leq j \leq t_{i}} L_{j}(S)$, where $L_{j}(S)$ is the language with words referring to valid event sequences generated during the execution of task $\tau_{j}$. By analogy, $L(T)=$ $\bigcup_{1 \leq i \leq n} \bigcup_{1 \leq j \leq t_{i}} L_{j}(T)$. Therefore, if $L_{j}(T) \subseteq L_{j}(S)$, $\forall 1 \leq j \leq t_{i}$ and $\forall 1 \leq i \leq n$ then also $L(T) \subseteq L(S)$. With this objective we prove that $L_{j}(T) \bigcap \overline{L_{j}(S)}=\emptyset$, $\forall 1 \leq j \leq t_{i}$ and $\forall 1 \leq i \leq n$. For a task $\tau_{j}$ to be schedulable all words in $L_{j}(T)$ must satisfy condition 5 . Similarly, all words in $L_{j}(S)$ must satisfy the condition $T_{\text {ready }} \leq d_{i}-$ wcet $_{j}-T_{\text {sw_wait }}$, where $T_{s w_{-} w_{a i t}}$ is the task waiting time in $T G S$ and $T_{s w_{-} \text {wait }_{j}} \leq T_{\text {wait }_{j}}$. Then the condition $L_{j}(T) \bigcap \overline{L_{j}(S)}=\emptyset$ becomes $\left(T_{\text {ready }}+m_{j} \leq\right.$ $d_{i}-$ wcet $_{j}-T_{\text {wait }_{j}}$ and $T_{\text {ready }}>d_{i}-$ wcet $\left._{j}-T_{\text {sw_wait }_{j}}\right)$. As from condition 3 we know that $m_{j} \geq 1$ and $T_{\text {ready }}$ cannot be at the same time higher than a value $\alpha$ and smaller than a value $\beta$ with $\alpha>\beta$, it follows $L_{j}(T) \bigcap \overline{L_{j}(S)}=\emptyset$ is true for any task $\tau_{j}$ and, therefore $L(T) \bigcap \overline{L(S)}=\emptyset$ holds.

\section{The H.264 Decoder: a CASE Study}

Using the proposed method for schedulability analysis, in this section we present a series of experiments in which we apply the proposed schedulability method to analyze the multimedia H.264 decoder [18]. We model the decoder as a component consisting of several MTTs. The tasks in each MTT and the precedence constraints between them are established from the workflow of the decoder as follows. During decoding each video frame is divided into slices and each slice is further split in blocks called macro-blocks (MB). The decoding process can be applied to several slices in parallel and consists of a well-defined set of steps. We define a MTT for each slice in a frame and each slice processing step is mapped to a task of the MTT. In the first stage of the process, numerical values are recovered from the binary codes of the compressed video (Entropy Decoding). Since a part of the data in the encoded video was computed through prediction, in the next stage (Dequantization and Inverse Transform DQIT) the differences between the predicted data and the real data are recovered. Next, in the motion compensation (or Inter-prediction) or Intra-prediction stage, each $\mathrm{MB}$ in the frame is decoded based on predicted data from previous frames or other MBs in the frame. Finally, the MBs of each slice are put together (Reconstruction) and a filtering stage is applied to improve quality of the decoded slice. Correspondingly, each MTT will have five tasks, one for each of the above stages. The MTT corresponding to the decoding process is presented in Figure 4. We consider that for each frame the same MTT will process the same slice.

To see how we can apply our method on the H.264 decoder, we extracted the execution parameters of the tasks in a MTT through profiling of the FFMPEG ${ }^{1}$ encoder using three real video files ${ }^{2}$ with increasing level of spatial details

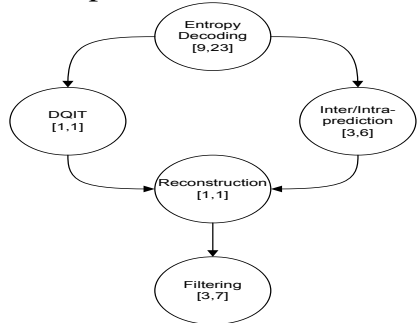

Figure 4. The MTT for slice decoding. and amount of movement. After profiling we have obtained the [bcet, wcet] intervals shown in Figure 4.

We have checked the schedulability of the component model using the UPPAAL model checker by issuing the verification of the $A[]$ not Error property. We ran experiments considering that for each frame of the video 2 (experiment 2-S), $3(3-\mathrm{S}), 4(4-\mathrm{S})$ or $5(5-\mathrm{S})$ slices are processed in parallel, where for each slice we have a MTT. The number of actual tasks in each experiment is five times the number of slices used for the experiment. First, for each task in a MTT, we have considered the time intervals in Figure 4 and for the period and deadline of the MTT we have chosen the value 100 . Since we noticed that the difference between the wcet and the bcet of each task and between execution times of different tasks influences the scalability of our method (the maximum number of supported MTTs), we then ran experiments in which all these time values were doubled (experiments 2-SD, 3-SD, 4-SD, 5-SD). In the last series of experiments (2-SD1, 3-SD1, 4-SD1, 5-SD1) we doubled only the execution time of the first task in the MTTs. We have chosen only this task since it has the greatest execution time, which varies along the largest interval. For all experiments we have used a set of three execution servers, one on each processor, with periods equal to 50 and a total processor utilization of 2.0 meaning that every 50 time units the servers provide 100 execution units to the decoder. The experiments were executed on a machine with Intel Core 2 Quad 2.40 GHz processor and 4 GB RAM running Ubuntu. The model checking time of all experiments is presented in Figure 5.

We can see in Figure 5 that although the number of tasks gets up to 25 the model checking time is rather

\footnotetext{
${ }^{1}$ http://ffmpeg.org/

${ }^{2}$ We have used the QCIF Akiyo, Foreman and Mobile videos from http://trace.eas.asu.edu/yuv
} 


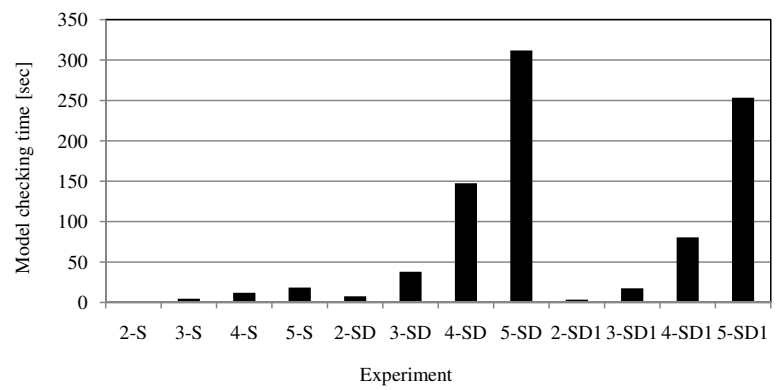

Figure 5. Model checking time

small (maximum 310 seconds). Also it can be seen that the complexity is a factor of the number of tasks, but is influenced also by the difference between the best and worst case execution time. Also, from the last four experiments (2-SD1 - 5-SD1) it can be observed that by increasing the difference between parameters of different tasks, the model checking time also grows even more than when we double all task parameters. We believe this is due to the fact that the change in these experiments has increased the nondeterminism of the model.

\section{CONClusions}

In this paper, we have proposed an approximation method for analysis of schedulability of component-based applications using model checking. Using timed automata we have provided a decidable method for schedulability verification in multi-core systems. The method captures dependencies between preemptable tasks and is able to capture continuous task execution times using a discrete time formalism. We have shown that the approximations in our method give a sufficient condition to determine the schedulability of a component and, by using iteratively the analysis on the parent application we can also prove the schedulability of the application. The applicability of our method was shown on a H.264 decoder.

\section{ACKNOWLEDGMENTS}

This research is supported by eMuCo, a European project supported by the European Union under the Seventh Framework Programme (FP7) for research and technological development.

\section{REFERENCES}

[1] A. Easwaran, I. Shin, and I. Lee, "Optimal virtual clusterbased multiprocessor scheduling," Real-Time Systems, vol. 43, no. 1, 2009.

[2] B. B. Brandenburg and J. H. Anderson, "Integrating hard/soft real-time tasks and best-effort jobs on multiprocessors," in ECRTS '07: Proceedings of the 19th Euromicro Conference on Real-Time Systems, 2007.

[3] F. Cassez and K. G. Larsen, "The impressive power of stopwatches," in CONCUR '00: Proceedings of the 11th International Conference on Concurrency Theory, 2000.
[4] Y. Kesten, A. Pnueli, J. Sifakis, and S. Yovine, "Decidable integration graphs," Information and Computation, vol. 150, no. $2,1999$.

[5] R. Graham, "Bounds on the performance of scheduling algorithm," SIAM Journal of Applied Mathematics, vol. 17, no. 2, pp. 416-429, 1969.

[6] P. Krcal, M. Stigge, and W. Yi, "Multi-processor schedulability analysis of preemptive real-time tasks with variable execution times," in FORMATS '07: Proceedings of the 5th International Conference on Formal Modeling and Analysis of Timed Systems, vol. 4763, October 2007, pp. 274-289.

[7] R. Alur and D. L. Dill, "A theory of timed automata," Theoretical Computer Science, vol. 126, no. 2, 1994.

[8] S. Sentilles, A. Pettersson, D. Nystrom, T. Nolte, P. Pettersson, and I. Crnkovic, "Save-IDE - A tool for design, analysis and implementation of component-based embedded systems," in ICSE '09: Proceedings of the 31st International Conference on Software Engineering, 2009.

[9] K. G. Larsen, P. Pettersson, and W. Yi, "UPPAAL in a nutshell," International Journal on Software Tools for Technology Transfer, vol. 2, no. 1, pp. 134-152, 1997.

[10] X. Ke, K. Sierszecki, and C. Angelov, "COMDES-II: A component-based framework for generative development of distributed real-time control systems,' in RTCSA '07: Proceedings of the 13th IEEE International Conference on Embedded and Real-Time Computing Systems and Applications, 2007.

[11] T. Amnell, E. Fersman, L. Mokrushin, P. Pettersson, and W. Yi, "TIMES - a tool for modelling and implementation of embedded systems," in TACAS '02: Proceedings of the 8th International Conference on Tools and Algorithms for the Construction and Analysis of Systems, 2002.

[12] N. Guan, Z. Gu, Q. Deng, S. Gao, and G. Yu, "Exact schedulability analysis for static-priority global multiprocessor scheduling using model-checking," in SEUS'07: Software Technologies for Embedded and Ubiquitous Systems, vol. 4761, September 2007, pp. 263-272.

[13] G. Madl, N. Dutt, and S. Abdelwahed, "A conservative approximation method for the verification of preemptive scheduling using timed automata," in RTAS '09: Proceedings of the 15th IEEE Real-Time and Embedded Technology and Applications Symposium, 2009.

[14] G. Lipari and E. Bini, "A methodology for designing hierarchical scheduling systems," Journal of Embedded Computing, vol. 1, no. 2, 2005.

[15] M. G. Harbour, "Architecture and contract model for processors and networks," Universidad de Cantabria, Technical Report D-AC1, 2006.

[16] G. Macariu and V. Cretu, "Model-based analysis of contractbased real-time scheduling," in SEUS'09: Software Technologies for Embedded and Ubiquitous Systems, vol. 5860, November 2009, pp. 227-239.

[17] T. A. Henzinger, P. W. Kopke, A. Puri, and P. Varaiya, "What's decidable about hybrid automata?" in STOC '95: Proceedings of the 27th annual ACM Symposium on Theory of Computing, 1995.

[18] "ISO/IEC 14496-10. coding of audio-visual objects. part 10: Advanced Video Coding." 